\title{
Acute Limb Ischemia Secondary to Catastrophic Massive Intracardiac Thrombus in Patient with Mitral Stenosis: A Case Report and Mini Literature Review
}

\author{
Rachmat Ageng Prastowo ${ }^{\mathrm{a}}$, Mohammad Budiarto ${ }^{\mathrm{a}}$, Yosua Hendriko Manurung ${ }^{\mathrm{a}}$ \\ rachmat.ageng18@gmail.com \\ ${ }^{a}$ Department of Cardiology and Vascular Medicine, Airlangga University - dr. Soetomo General Academic Hospital, Surabaya, \\ Indonesia
}

\begin{abstract}
Mitral stenosis is a common long-term complication of rheumatic fever and can result in left atrial (LA) enlargement, increasing the risk of thrombus formation. LA thrombi were associated with an increased risk of embolic events such as acute limb ischemia (ALI). ALI occurs when arterial flow to an extremity is abruptly interrupted, which may result in amputation or death. A thrombotic or embolic arterial occlusion is typically the cause of ALI. The authors describe a case of massive thrombus in the left atrium associated with mitral stenosis, which resulted in a systemic thromboembolic complication. A 51-year-old woman with chronic mitral stenosis was hospitalized for palpitation and limb pain without dyspnea or chest pain. Atrial fibrillation was detected via electrocardiography (AF). Echocardiography revealed a rheumatic, thickened, and stenotic mitral valve, as well as a $30 \times 24 \mathrm{~mm}$ thrombus in the left atrium. She was successfully treated with thrombus removal and mitral valve replacement. Postoperative complications were minimal, and she was discharged from the hospital four days after surgery.
\end{abstract}

Keywords: Mitral stenosis; Left atrial thrombus; Acute limb ischemia

\section{Introduction}

Left atrial thrombus formation is a serious complication of severe mitral stenosis $(26 \%-33 \%$ in severe stenosis) and atrial fibrillation $(\mathrm{AF})^{1-3}$. There are many factors that can result in left atrial thrombus such as atrial fibrillation, the size of left atrium, older age, and the severity of the mitral stenosis, which has significant complication resulting in stroke, systemic emboli, and sudden death ${ }^{4}$. These thrombo-emboli complications have higher rates in patient with rheumatic mitral stenosis, especially patients with AF. However, mitral rheumatic patients with sinus rhythm also have risk in forming of clot in left atrial ${ }^{4,5}$.

Anticoagulant therapy is the first line of treatment for left atrial thrombus. Even so, if first-line therapy fails or if the thrombus has expanded in the left atrium, more aggressive therapy such as thrombolysis and surgical intervention may be required ${ }^{3}$. Serial transesophageal echocardiogram evaluation is needed for following up the given therapies and also for making sure the complete resolution of the thrombus.

Acute limb ischemia (ALI) is a potential complication of systemic emboli in patients with mitral stenosis. The majority of ALIs ( $85 \%$ ) are caused by in situ thrombosis, while $15 \%$ are caused by arterial emboli occlusion Over $90 \%$ of emboli are formed in the heart ${ }^{6}$ and are typically caused by AF or acute myocardial infarction. Emboli frequently adhere at the bifurcation of arteries, such as aortoiliac, femoral, and distal popliteal artery ${ }^{6}$. 
In this case, we report a case of systemic embolus in the form of ALI in a patient with mitral stenosis and left atrial thrombus and AF complications.

\section{Case}

A 51-year-old woman admitted to the emergency room with palpitation since yesterday. This complaint was not accompanied with shortness of breath nor chest pain. In addition to the palpitation, she also complained of pain in her leg which had been felt in the past 4 days before admission which accompanied with numbness and tingling sensations. She had a history of mitral stenosis which had been diagnosed since 2015 with AF complication which diagnosed around 2018-2019. She was prescribed Furosemide $40 \mathrm{mg}$ bid, spironolactone $50 \mathrm{mg}$ od, bisoprolol $2.5 \mathrm{mg}$ od, and warfarin $2 \mathrm{mg}$ od on a regular basis at the cardiology outpatient clinic. She admitted that she had not been taking warfarin for the last 12 days because she had more menstrual blood loss than usual. She had no smoking habit, and no history of diabetes mellitus nor hypertension.

When admitted, she had 100/60 mmHg blood pressure, an irregular pulse at $140 \mathrm{bpm}$, a respiratory rate of 23 times per minute, body temperature at 36.8 degrees Celsius, and 98 percent of peripheral oxygen saturation. She looked slight anemic for the eye conjunctiva observation. We found irregular S1 and S2 at heart auscultation and the murmur was hard to be evaluated. At further examination, we found the left lower extremity was cold, pale, and decreasing pulsation of popliteal artery until dorsalis pedis artery. There is no way to determine the oxygen saturation of all toes.

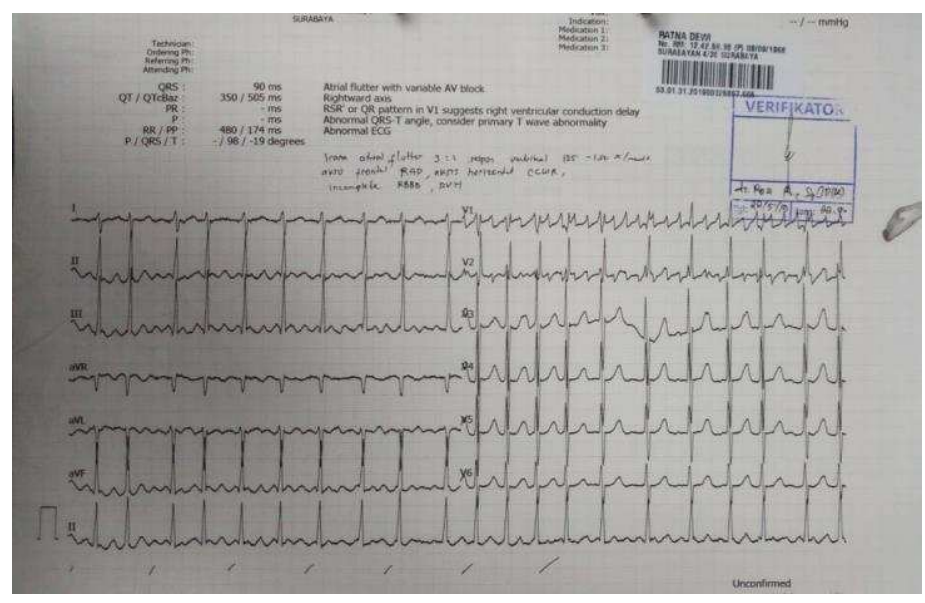

Fig. 1. ECG when the patient admitted at ER department

The only abnormal laboratory finding was patient's Hemoglobin at 9.8. We discovered atrial fibrillation with a rapid ventricular response of 125 to 150 beats per minute on the electrocardiogram, and chest $\mathrm{x}$-ray showed mitral heart configuration with $60 \%$ of cardiac thoracic ratio (CTR). Transthoracic echocardiography was performed and we found severe mitral stenosis with left atrial thrombus measured at $3.2 \times 4.0 \mathrm{~cm}$ which had not found from the TTE evaluation a year before. From the Doppler ultrasound (DUS) at the left lower extremity was found monophasic wave at left femoral common artery and no flow at all of the distal parts. 


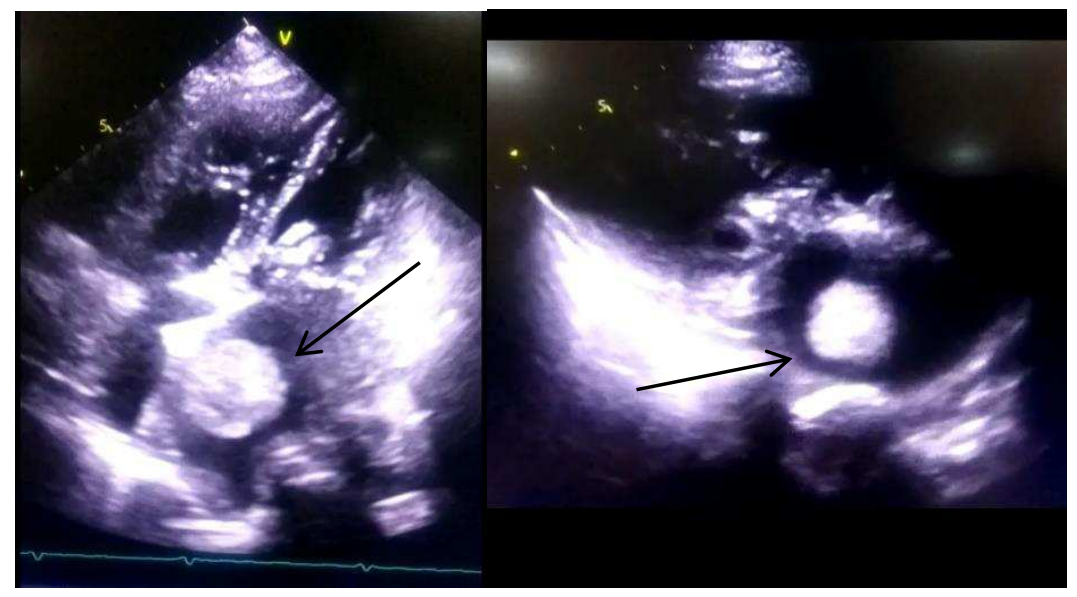

Fig. 2. Left atrial thrombus shown in TTE evaluation (black arrow)

Judging from the clinical conditions, ECG, and radiography findings, it was decided to perform fast digitalization with digoxin $0.25 \mathrm{mg}$ intravenous, anticoagulant with heparin starting at $18 \mathrm{unit} / \mathrm{kg} / \mathrm{hour}$ intravenous, and immediate arteriography. From arteriography we found total occlusion at the left femoral common artery with thrombus finding with 5 to $6 \mathrm{~cm}$ in length.

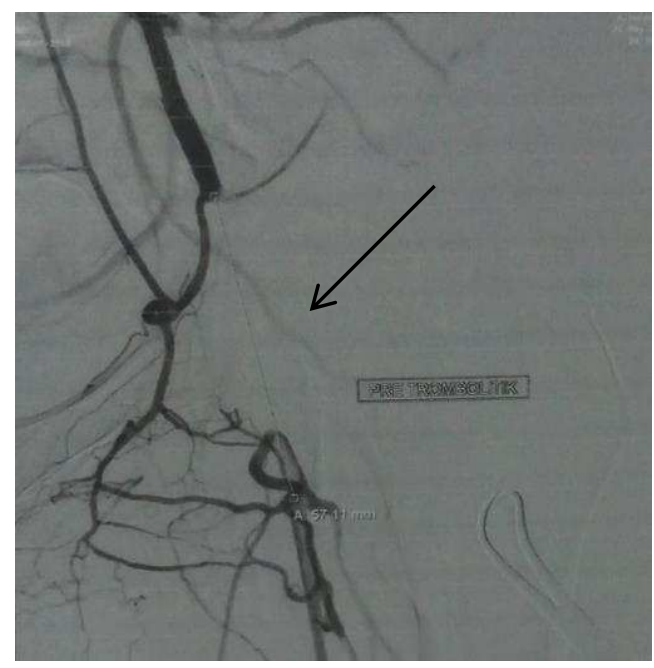

Fig. 3. Arteriography showed a filling defect (total occlusion) of the left femoral common artery (black arrow)

We performed catheter direct thrombolysis (CDT) with 200,000 units of streptokinase in 60 minutes intraarterial in left femoral common artery. When the streptokinase had been administered around 80.000 units, the patient complained having her chest felt being crushed, her vital signs were normal at that moment. We stopped the streptokinase and consult the Thoracic and Cardiovascular Surgery Department and decided to perform 
thrombectomy urgent. The procedure accomplished anterogradely as far as $40 \mathrm{~cm}$ and $8 \mathrm{~cm}$ thrombus was found, retrogradely $30 \mathrm{~cm}$ and found $2 \mathrm{~cm}$ thrombus. Then retrogradely from the procedure was found thrombus with 5 to $6 \mathrm{~cm}$ in length.

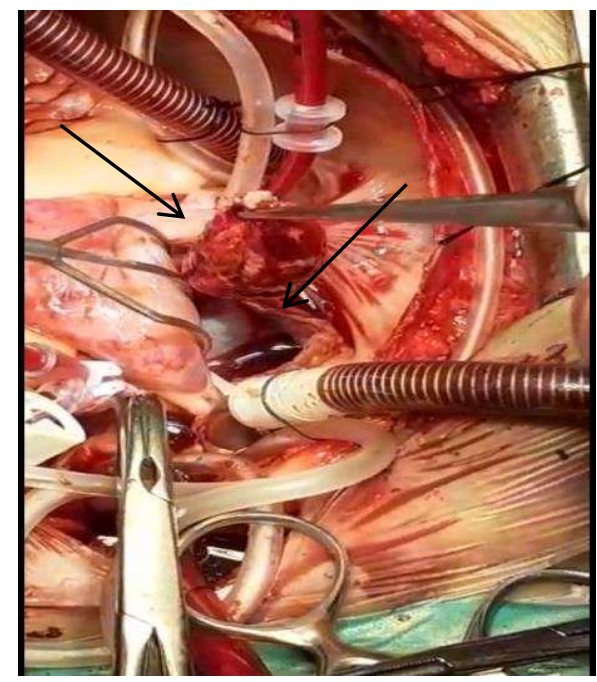

Fig. 4. Evacuation process of left atrial thrombus (black arrow)

A week after the ALI treatment, the patient was operated to undergo mitral valve replacement (MVR) and left atrial thrombus evacuation. The procedure was completed successfully. Four days after MVR operation, the patient was discharged. DUS evaluation showed improvement of arteries of the left lower extremity, in which we found triphasic waves in profound femoral artery, popliteal artery, anterior tibialis artery, and posterior tibialis artery.

\section{Discussion}

The underlying disease in this case is mitral stenosis which mostly found in Rheumatic Heart Disease (RHD). Although the incidence in developed countries has decreased, RHD remains one of major problems in developing countries. The excessive dilatation of the left atrium (greater than $65 \mathrm{~mm}$ ), which also known as gigantic atrium, is the most pathological structural findings in rheumatic mitral stenosis. Additionally, severe mitral valve prolapse, persistent atrial fibrillation, and a heart defect can complicate this condition. ${ }^{7-9}$ Atrium dilatation is a compensatory mechanism induced by increased intracavity pressure in order to prevent lung edema and decrease lung failure. ${ }^{10}$ Atrial fibrillation was triggered by dilation and fibrosis in the left atrium, which increased the risk of systemic thromboembolism and enlarging the left atrium further. ${ }^{11}$

Atrial fibrillation, which affects $40 \%$ of patients, can exacerbate mitral stenosis and act as a risk factor for thromboembolic events in patients with mitral stenosis. ${ }^{12}$ The stagnant blood flow in the left atrial appendage (LAA) and the atrium's lack of maximal contractility during fibrillation increase the risk of arterial thromboembolism in patients with mitral stenosis (MS).,13-15 This happens in our patient, forming systemic thrombus after having history of MS which had been diagnosed since 2015, worsened by AF which diagnosed since 2018. The other prognosis in MS patient can be a thromboembolic stroke which can lead to neurological deficit. $^{16}$ 
Patients with severe MS and AF, as shown from our patient, have incidence risk of thromboembolic events, which need anticoagulant therapy. The levels of fibrinopeptide A, thrombin-antithrombin III complex, and von Willebrand factor in the left atrium increase in patients with MS. ${ }^{17}$ The coagulation system has been demonstrated to be more active in patients with mitral stenosis complicated by left atrial enlargement, requiring adequate anticoagulant therapy. Enlargement of the left atrium has been linked to increased blood flow turbulence, and regardless of anticoagulant therapy, the risk of thromboembolism increases with left atrium enlargement. ${ }^{18}$ As evidenced by our patient, despite prior warfarin treatment, she developed thromboembolic complications.

One of thromboembolic events, which happened as complication of MS with AF in our patient, is Acute Limb Ischemia. ALI is a rapidly deteriorating limb perfusion condition that poses a threat to the tissue and requires immediate evaluation. Patients are included in ALI if the symptoms show before 2 weeks. After 2 weeks, it is called chronic limb ischemia. ${ }^{19-22}$ Embolism, arterial thrombosis, peripheral artery aneurysm, dissection, and arterial trauma are the most frequent causes of ALI. As in our patient was likely caused because of embolism and arterial thrombosis which developed in her left atrium and her lower limb vessel.

The degree of ischemia is grouped according to the Rutherford classification. The assessment is based on the viability of the damaged limb tissue. Occasionally, it is difficult to distinguish between classes IIa and IIb, as well as between classes IIb and III. Immediate diagnosis and revascularization using thrombolytics and/or catheter-based thrombus aspiration or by surgery can reduce the chance of limb death. Patients with irreversible ischemia should undergo amputation (Class III). Although revascularization can be performed, patients with ALI have a high mortality and amputation rate. ${ }^{19}$ Over the previous century, the general etiology of embolization has shifted away from rheumatic heart disease or congenital heart disease in relatively young individuals and toward cardiac arrhythmias or in situ thrombosis in the elderly. It is critical to remember that ALI caused by arterial thrombosis or atherosclerotic embolization of vessels is becoming more prevalent, which has significant implications for its treatment and management. ${ }^{23,24}$

ALI is a serious disease that can result in amputation of limbs. The decision to determine the best treatment and therapy becomes very important. Often the limb cannot be saved because of irreversible ischemia, and amputation is necessary to save the patient's life. Therefore, appropriate treatment for ALI should be initiated immediately to prevent worsening of limb ischemia and other severe complications. The clinical manifestations of ALI vary according to the location and duration of the arterial occlusion, the presence of collateral circulation, and the metabolic changes caused by tissue ischemia. Frequently, following arterial occlusion, signs of ischemia manifest at a level/joint distal to the occlusion site.

It is sometimes difficult to define the clinical difference between acute and thrombotic embolic occlusion. Acute on CLI patients frequently have a history of intermittent claudication and risk factors for PAD such as smoking, hypertension, renal impairment, and diabetes. The clinical assessment must incorporate the patient's history. Symptoms of ALI that appear suddenly are characteristic of arterial embolism. Patients who develop ALI as a result of thrombosis will exhibit gradual symptoms associated with preexisting PAD and increased collateral circulation. The vast majority of embolic occlusions are due to dysrhythmias, with two-thirds caused by AF, and $20 \%$ by ventricular thrombus (which can have a variety of causes) ${ }^{25}$. Valvular heart disease remains a common cause of ALI in low- and middle-income countries.

The classic "6Ps" (pain, pallor, pulselessness, poikilothermia, paresthesia, and paralysis) can aid in determining the clinical significance of ischemia. However, except in the most severe cases of ALI with previously normal arteries, these symptoms do not always manifest in daily practice. Peripheral pulse detection can be improved by calculating the ankle brachial index (ABI) or using Doppler ultrasound. ${ }^{26,27}$ The ABI examination rate in ALI cases is also a predictor of patient's prognosis where the index $<0.7$ represents a critical condition of perfusion. ${ }^{28}$ Sensory and motor dysfunction are indicators of a threatened limb in urgent need of 
revascularization. The Rutherford classification of ALI is the most often used method for determining whether an ischemic limb may be saved and for guiding therapeutic care methods.

Digital subtraction angiography (DSA) remains the gold standard examination for ALI in terms of diagnostic accuracy.$^{29}$ DSA can help define the etiology and has the added benefit of allowing for concurrent treatment strategies. This should be explored in conjunction with endovascular surgical management in clinical practice. Embolic occlusion is defined by the presence of a crescent-shaped occlusion, or meniscus sign, in combination with the normal appearance of the remaining vessels. Thrombotic occlusions are distinguished by the presence of additional areas of atherosclerosis and the formation of multiple collaterals. DSA should be accessed via an arterial route that allows for the evaluation of both inflow and outflow. Intra-arterial vasodilators can be used to alleviate vasospasm in vessels distal to the occlusion site and thus improve visualization of the further arterial vascular bed. ${ }^{30}$

There is a dearth of data on duplex ultrasound (DUS) diagnostic accuracy in the ALI setting. DUS has an 88 percent sensitivity ( 95 percent confidence interval [CI] 80 percent -98 percent) and a 96 percent specificity (95 percent CI 89 percent -99 percent) for detecting $>50$ percent stenosis or occlusion in patients with chronic occlusion. ${ }^{31}$ DUS is accurate for detecting total or subtotal obstruction of the common femoral, superficial femoral, popliteal, and bypass graft arteries in $90 \%$ of cases where revascularization is considered, including patients with ALI. ${ }^{32}$ Although the diagnostic accuracy is lower when detecting tibial artery stenosis or occlusion, such distal lesions are uncommon causes of ALI. As such, DUS should not be used exclusively to rule out arterial occlusion.

Computable tomography angiography is another imaging modality (CTA). To obtain a clear view of the leg's arteries, CTA requires the administration of non-ionic contrast. Although there is a link between iodized contrast use and acute kidney injury, it is a disproportionate relative concern when dealing with a potentially fatal condition. Current CTA technology enables coverage of the entire body in a single acquisition, with fast acquisition times, high resolution, and the possibility of reconstructing axial images with similar accuracy to DSA images. ${ }^{33}$ CTA has the upper hand of allowing for evaluation of the thoracic and abdominal aorta for possible embolism sources, as well as the bowel vessels for other emboli. Extravascular abnormalities may be observed that are related to the pathogenesis of ALI or have significant clinical implications.

DSA, CTA, and DUS can all be contemplated for imaging in patients with ALI, depending on expert presence, availability over time, and patient preference. CTA is the most frequently used imaging modality due to its availability, and it should be used for treatment planning unless the ischemia is severe enough that additional imaging is not possible.

The primary goal of management of ALI is reperfusion of the ischemic limb. The initial medical treatment for ALI is to administer adequate analgesics and intravenous unfractionated heparin (UFH) at a dose of 5,000 units, or 70-100 units/kg body weight, followed by a follow-up infusion dose adjusted according to the patient's reaction and monitored with activated partial thromboplastin time (APTT). The objectives of this early therapy are to prevent emboli from spreading and to produce an anti-inflammatory effect. ${ }^{34}$

The urgency with which treatment and reperfusion measures are initiated is determined by the severity of limb ischemia as determined by the Rutherford clinical classification. The management strategy based on the widely published Rutherford classification ${ }^{19}$ incorporates angiography, endovascular techniques, and surgical techniques. Various revascularization methods are available, such as surgical thrombo-embolectomy, bypass, catheter-directed thrombolysis (CDT), thrombus aspiration/ mechanical thrombectomy (with or without thrombolysis) and hybrid procedures including thrombendarteriectomy. The approach, which will be used, is determined by a variety of factors, including expert staff, available team facilities, as well as patient characteristics such as the length and the degree of severity of ALI, the site and origin of blockage, comorbidities, and potential therapeutic risks. 
Class I limb ischemia does not pose a threat requiring immediate action, and may frequently managed as chronic limb ischemia, based on a complete vascular examination. Most of these individuals actually have multiple chronic conditions, making conservative approaches more appropriate. ${ }^{35}$ However, some grade 1 ischemic patients warrant revascularization and are managed similarly to class IIA patients, if the limb is at risk. Therefore, immediate angiography is a necessary initial step to salvage the limb by endovascular techniques such as CDT or percutaneous mechanical thrombectomy (PMT).

Leg ischemia that threatens tissue perfusion, Rutherford classification class IIB, can still be saved if revascularization can be done immediately. Surgical embolectomy is the most frequently used procedure for rapidly reperfusing the limb and ensuring tissue continuity. Embolic occlusive lesions may just necessitate an embolectomy operation, whereas thrombotic occlusion may necessitate endarterectomy or bypass surgery. As is the case with endovascular procedures, assessment arteriography is often suggested unless embolectomy restores the distal pulse to normal. ${ }^{35}$ There is widespread agreement that evaluation angiography following thrombo-embolectomy is necessary to document the procedure's success, as residual thrombi are common and their detection is associated with a decreased risk of re-intervention and limb amputation. ${ }^{36,37}$ Re-embolectomy or bypass surgery may be considered if residual thrombus is discovered following embolectomy. Another possibility is to inject an intraoperative thrombolytic agent directly into the associated artery in order to dissolve any remaining thrombus..$^{38-40}$

Ischemia of Ruhterford class III is presumed to be a permanent state. Along with audible vessel Doppler pulse examination, the patient had severe sensory and motor loss, along with muscle rigidity and persistent skin discoloration. Evaluation of the duration of ischemia is very important in this case. If the disease is identified quickly (within two hours of the onset of ischemia), immediate embolectomy can still save the limb. Efforts at revascularization exceeding 2 hours are unlikely to be successful, and can lead to renal failure due to rhabdomyolysis and contractures of adjacent muscles, which may interfere with amputation at a later date. In patients with severe ischemia, compartment syndrome frequently occurs in conjunction with surgical reperfusion strategies. Swelling pressure exceeding $30 \mathrm{mmHg}$ after revascularization is an indication that fasciotomy is necessary. ${ }^{41}$

A reperfusion syndrome may emerge following revascularization and reperfusion of the affected limb. Activated leukocytes and platelets from reperfusion stimulate the production of inflammatory mediators, which disrupt the cellular membrane ion pumps, resulting in the generation of free radicals and cell death. ${ }^{42}$ As consequence of this, myoglobin, potassium, lactate, and microthrombi are all absorbed into the systemic circulation. This can result in kidney failure, arrhythmias, and, in severe cases, death. This condition is related with morbidity and mortality rates up to $41 \%{ }^{43}$

Anticoagulation is not always necessary, depending on the cause of limb ischemia. Prolonged anticoagulation should be given to patients with uncorrected cardiac or other embolic sources. Repeated embolism occurs at a rate of between $6 \%$ and $45 \%$. Another research also found the risk of relapse of $9 \%$ in patients whom received anticoagulant and $31 \%$ whom are not given. ${ }^{44}$

\section{Conclusion}

ALI is a medical emergency that can threaten life and limb death, where embolism is one of the most prevalent causes of ALI. AF arrhythmia anomalies in MS patients cause intracardiac thrombus development, which increases the risk of systemic embolism, including ALI, if proper anticoagulant medication is not provided. Comprehensive management is needed in dealing with MS cases that cause ALI, to get good treatment results for patients. Along with the development of diagnostic and therapeutic modalities in the management of ALI, it is hoped that patient management will be better, so that the incidence of amputation and death can be avoided. 


\section{References}

1. Conradie C, Marx JD, Schall R. Left atrial size-A risk factor for left atrial thrombi in mitral stenosis. Clin Cardiol. 1995;18(9). doi:10.1002/clc.4960180907

2. Bollmann A, Husser D, Stridh M, et al. Atrial fibrillatory rate and risk of left atrial thrombus in atrial fibrillation. Europace. 2007;9(8). doi:10.1093/europace/eum125

3. Srimannarayana J, Varma RS, Satheesh S, Anilkumar R, Balachander J. Prevalence of Left Atrial Thrombus in Rheumatic Mitral Stenosis with Atrial Fibrillation and Its Response to Anticoagulation: A Transesophageal Echocardiographic Study. Indian Heart J. 2003;55(4).

4. Acartürk E, Usal A, Demir M, Akgül F, Özeren A. Thromboembolism risk in patients with mitral stenosis. Jpn Heart J. 1997;38(5). doi:10.1536/ihj.38.669

5. Shrestha NK, Moreno FL, Narciso F V., Torres L, Calleja HB. Two-dimensional echocardiographic diagnosis of left atrial thrombus in rheumatic heart disease. A clinicopathologic study. Circulation. 1983;67(2). doi:10.1161/01.CIR.67.2.341

6. Walker TG. Acute Limb Ischemia. Tech Vasc Interv Radiol. 2009;12(2). doi:10.1053/j.tvir.2009.08.005

7. Plaschkes J, Borman JB, Merin G, Milwidsky H. Giant left atrium in rheumatic heart disease: a report of 18 cases treated by mitral valve replacement. Ann Surg. 1971;174(2). doi:10.1097/00000658-197108000-00004

8. Hurst JW. Memories of patients with a giant left atrium. Circulation. 2001;104(22). doi:10.1161/hc4701.100775

9. Schwammenthal E, Vered Z, Agranat O, Kaplinsky E, Rabinowitz B, Feinberg MS. Impact of atrioventricular compliance on pulmonary artery pressure in mitral stenosis: An exercise echocardiographic study. Circulation. 2000;102(19 SUPPL.). doi:10.1161/01.CIR.102.19.2378

10. Kawazoe K, Beppu S, Takahara Y, et al. Surgical treatment of giant left atrium combined with mitral valvular disease. Plication procedure for reduction of compression to the left ventricle, bronchus, and pulmonary parenchyma. J Thorac Cardiovasc Surg. 1983;85(6). doi:10.1016/s0022-5223(19)37479-3

11. Hodzic E, Granov N. Gigantic Thrombus of the Left Atrium in Mitral Stenosis. Med Arch (Sarajevo, Bosnia Herzegovina). 2017;71(6). doi:10.5455/medarh.2017.71.449-452

12. Carabello BA. Modern management of mitral stenosis. Circulation. 2005;112(3). doi:10.1161/CIRCULATIONAHA.104.532498

13. Mahmood ul Hassan, Hussain C, Gul AM, ullah Jan H, Hafizullah M. Frequency of left atrial and appendage clot in patients with severe mitral stenosis. J Ayub Med Coll Abbottabad. 2010;22(2).

14. Goswami KC, Yadav R, Rao MB, Bahl VK, Talwar KK, Manchanda SC. Clinical and echocardiographic predictors of left atrial clot and spontaneous echo contrast in patients with severe rheumatic mitral stenosis: A prospective study in 200 patients by transesophageal echocardiography. Int J Cardiol. 2000;73(3). doi:10.1016/S0167-5273(00)00235-7

15. Goswami KC, Yadav R, Bahl VK. Predictors of left atrial appendage clot: A transesophageal echocardiographic study of left atrial appendage function in patients with severe mitral stenosis. Indian Heart J. 2004;56(6).

16. Pourafkari L, Ghaffari S, Bancroft GR, Tajlil A, Nader ND. Factors associated with atrial fibrillation in rheumatic mitral stenosis. Asian Cardiovasc Thorac Ann. 2015;23(1). doi:10.1177/0218492314530134

17. Ates M, Sensoz Y, Abay G, Akcar M. Giant left atrium with rheumatic mitral stenosis. Texas Hear Inst J. 2006;33(3).

18. Sanfilippo AJ, Abascal VM, Sheehan M, et al. Atrial enlargement as a consequence of atrial fibrillation. A prospective echocardiographic study. Circulation. 1990;82(3). doi:10.1161/01.CIR.82.3.792

19. Rutherford RB, Baker JD, Ernst C, et al. Recommended standards for reports dealing with lower extremity ischemia: Revised version. J Vasc Surg. 1997;26(3). doi:10.1016/S0741-5214(97)70045-4

20. Norgren L, Hiatt WR, Dormandy JA, Nehler MR, Harris KA, Fowkes FGR. Inter-Society Consensus for the Management of Peripheral Arterial Disease (TASC II). J Vasc Surg. 2007;45(1 SUPPL.). doi:10.1016/j.jvs.2006.12.037

21. Aboyans V, Ricco JB, Bartelink MLEL, et al. 2017 ESC Guidelines on the Diagnosis and Treatment of Peripheral Arterial Diseases, in collaboration with the European Society for Vascular Surgery (ESVS). Eur Heart J. 2018;39(9). doi:10.1093/eurheartj/ehx095

22. Conte MS, Bradbury AW, Kolh P, et al. Global Vascular Guidelines on the Management of Chronic Limb-Threatening Ischemia. Eur J Vasc Endovasc Surg. 2019;58(1). doi:10.1016/j.ejvs.2019.05.006

23. Ljungman C, Adami H -O, Bergqvist D, Sparen P, Bergström R. Risk factors for early lower limb loss after embolectomy for acute arterial occlusion: A population-based case-control study. Br J Surg. 1991;78(12). doi:10.1002/bjs.1800781224

24. Acosta S, Kuoppala M. Update on intra-arterial thrombolysis in patients with lower limb ischemia. J Cardiovasc Surg (Torino). 2015;56(2).

25. Santistevan JR. Acute Limb Ischemia: An Emergency Medicine Approach. Emerg Med Clin North Am. 2017;35(4). doi:10.1016/j.emc.2017.07.006

26. Bailey MA, Griffin KJ, Scott DJA. Clinical assessment of patients with peripheral arterial disease. Semin Intervent Radiol. 2014;31(4). doi:10.1055/s-0034-1393964

27. Kuoppala M, Åkeson J, Acosta S. Outcome after thrombolysis for occluded endoprosthesis, bypasses and native arteries in patients with lower limb ischemia. Thromb Res. 2014;134(1). doi:10.1016/j.thromres.2014.02.030

28. Plate G, Jansson I, Forssell C, Weber P, Oredsson S. Thrombolysis for Acute Lower Limb Ischaemia-A Prospective, Randomised, Multicentre Study Comparing Two Strategies. Eur J Vasc Endovasc Surg. 2006;31(6). doi:10.1016/j.ejvs.2005.11.017

29. Weiss CR, Azene EM, Majdalany BS, et al. ACR Appropriateness Criteria® Sudden Onset of Cold, Painful Leg. J Am Coll 
Radiol. 2017;14(5). doi:10.1016/j.jacr.2017.02.015

30. Jaffery Z, Thornton SN, White CJ. Acute limb ischemia. Am J Med Sci. 2011;342(3). doi:10.1097/MAJ.0b013e31820ef345

31. Collins R, Burch J, Cranny G, et al. Duplex ultrasonography, magnetic resonance angiography, and computed tomography angiography for diagnosis and assessment of symptomatic, lower limb peripheral arterial disease: Systematic review. Br Med J. 2007;334(7606). doi:10.1136/bmj.39217.473275.55

32. Hingorani AP, Ascher E, Marks N, et al. Limitations of and lessons learned from clinical experience of 1,020 duplex arteriography. Vascular. 2008;16(3). doi:10.2310/6670.2008.00014

33. Madhuripan N, Mehta P, Smolinski SE, Njuguna N. Computed Tomography Angiography of the Extremities in Emergencies. Semin Ultrasound, CT MRI. 2017;38(4). doi:10.1053/j.sult.2017.03.001

34. Gerhard-Herman MD, Gornik HL, Barrett C, et al. 2016 AHA/ACC guideline on the management of patients with lower extremity peripheral artery disease: A report of the American college of cardiology/American Heart Association Task Force on Clinical Practice Guidelines. Circulation. 2017;135(12). doi:10.1161/CIR.0000000000000471

35. Goldstein JA, Mishkel G. Choosing the correct therapeutic option for acute limb ischemia. Interv Cardiol. 2011;3(3). doi:10.2217/ica.11.28

36. Lipsitz EC, Veith FJ. Fluoroscopically assisted thromboembolectomy: Should it be routine? Semin Vasc Surg. 2001;14(2). doi:10.1053/svas.2001.23165

37. Zaraca F, Stringari C, Ebner JA, Ebner H. Routine Versus Selective Use of Intraoperative Angiography During Thromboembolectomy for Acute Lower Limb Ischemia: Analysis of Outcomes. Ann Vasc Surg. 2010;24(5). doi:10.1016/j.avsg.2009.12.006

38. Knaus J, Ris HB, Do D, Stirnemann P. Intraoperative catheter thrombolysis as an adjunct to surgical revascularisation for infrainguinal limb-threatening ischaemia. Eur J Vasc Surg. 1993;7(5). doi:10.1016/S0950-821X(05)80361-5

39. Beard JD, Nyamekye I, Earnshaw JJ, Scott DJA, Thompson JF. Intraoperative streptokinase: A useful adjunct to balloon-catheter embolectomy. Br J Surg. 1993;80(1). doi:10.1002/bjs.1800800108

40. Witz M, Korzets Z, Ellis M, Shnaker A, Lehmann J. Intraoperative intra-arterial urokinase therapy after failed embolectomy in acute lower limb ischemia. J Cardiovasc Surg (Torino). 2002;43(6).

41. O'Connell JB, Quiñones-Baldrich WJ. Proper Evaluation and Management of Acute Embolic versus Thrombotic Limb Ischemia. Semin Vasc Surg. 2009;22(1). doi:10.1053/j.semvascsurg.2008.12.004

42. Beyersdorf F, Schlensak C. Controlled Reperfusion after Acute and Persistent Limb Ischemia. Semin Vasc Surg. 2009;22(1). doi:10.1053/j.semvascsurg.2009.01.005

43. Trummer G, Brehm K, Siepe M, Heilmann C, Schlensak C, Beyersdorf F. The management of acute limb ischemia. Minerva Chir. 2010;65(3).

44. Green RM, DeWeese JA, Rob CG. Arterial embolectomy before and after the Fogarty catheter. Surgery. 1975;77(1). doi:10.5555/uri:pii:0039606075900045 